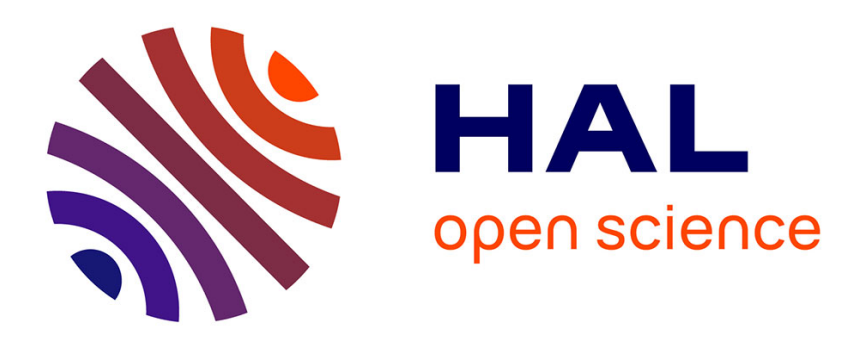

\title{
Non-additive interactions of nucleobases in model dinucleotide steps occurring in B-DNA crystals
}

Piotr Cysewski

\section{To cite this version:}

Piotr Cysewski. Non-additive interactions of nucleobases in model dinucleotide steps occurring in B-DNA crystals. Journal of Molecular Modeling, 2010, 16 (11), pp.1721-1729. 10.1007/s00894-0100722-8 . hal-00591241

\author{
HAL Id: hal-00591241 \\ https://hal.science/hal-00591241
}

Submitted on 8 May 2011

HAL is a multi-disciplinary open access archive for the deposit and dissemination of scientific research documents, whether they are published or not. The documents may come from teaching and research institutions in France or abroad, or from public or private research centers.
L'archive ouverte pluridisciplinaire HAL, est destinée au dépôt et à la diffusion de documents scientifiques de niveau recherche, publiés ou non, émanant des établissements d'enseignement et de recherche français ou étrangers, des laboratoires publics ou privés. 


\section{Editorial Manager(tm) for Journal of Molecular Modeling Manuscript Draft}

Manuscript Number: JMM01145R1

Title: Non-additive interactions of nucleobases in model dinucleotide steps occurring in B-DNA crystals

Article Type: Special Issue Prague

Keywords: many-body, intermolecular interactions, dinucleotide steps, 8-oxoguanine, B-DNA

Corresponding Author: Prof. Piotr Cysewski, Ph.D.

Corresponding Author's Institution: Collegium Medicum, Nicolaus Copernicus University

First Author: Piotr Cysewski, Ph.D.

Order of Authors: Piotr Cysewski, Ph.D.

Manuscript Region of Origin: POLAND

Abstract: Non-additivity of base-base interactions in all ten possible model dinucleotide steps were analyzed on MP2/aug-cc-pvDZ quantum chemistry level. Selected conformations of four nucleobases exactly matched to ones occurring in B-DNA crystals. In most of 162 analyzed tetramers both threeand four-body contributions are negligible except of $d(G p G)$ steps. However, in these dinucleotides both contributions are always of opposite signs and in all cases the sum of all non-additive part of intermolecular interactions do not exceed $2.6 \mathrm{kcal} / \mathrm{mol}$. This stands for less than $5 \%$ of the overall binding energy of dinucleotide steps. Also replacements of guanine with 8-oxoguanine in $\mathrm{d}(\mathrm{GpG})$ systems introduces non-additivity of the same magnitude as for canonical dinucleotides. It is observed linear relationships between values of the total binding energy obtained in the tetramer basis set and estimated energy exclusively in dimers basis sets with assumption of pairwise additivities. For all analyzed dinucleotides steps there are also linear correlations between amount of non-additive contributions and magnitude of pairs interactions. Based on differences in electrostatic contribution to the total binding energy of four nucleobases and polarity of dinucleotide steps three distinct classes of dinucleotide steps were identified.

Response to Reviewers: 1) I recommend to do a careful proofreading, there are many typos and some sentences are difficult to understand.

Thank you. Careful proofreading was applied and text was modified accordingly.

2) Page 3, Results and discussion

Alltogether 162 dinucleotide steps were analyzed. The number of individual steps is variable, why is it so? Why there was $48 \mathrm{~d}(\mathrm{GpG})$ steps, but only $10 \mathrm{~d}(\mathrm{GpC})$ steps? What were the PDB codes of structures from which steps were chosen, and which steps (chain ID, res ID) were chosen? What was the resolution of these structures? This information could go to the Supplementary material.

In supplementary material details related to analyzed structures are provided now. The differences in dinucleotide populations have strictly technical reason. Since in my previous work accepted for publication in Int.J.Quantum Chem. (DOI:10.1002/qua.22435) detailed analysis of d(GpG) steps were presented I have just included these data in this work. Consequently this dinucleotide is 
overrepresented. Since it constitutes its own class I do not think that such overrepresentation changes papers conclusions. On the other hand it is not necessary extending the number of other dinucleotide steps since the non-additivity is quite small and they were selected from broad range of data according to IIE and structural parameters diversities.

3) Page 3, Results and discussion

The numbers of steps are large enough to perform some statistical analysis of resulting energies. E.g. ANOVA could be performed to test whether the mean energies in dinucleotide steps differ significantly each from other. Also, is the distribution of energies for individual steps Gaussian or not? If not, some non-parametric testing procedures could be used. In general, the number of data seems to me to be large enough to do their thorough statistical analysis, which would considerably improve the quality of the results, maybe even leading to some yet unnoticed conclusions.

In fact I did statistical analysis but intentionally data were not discussed in details. The differences in the median values between all pairs of analyzed dinucleotide classes are greater than would be expected by chance $(\mathrm{P}=<0.001)$. Hence all three dinucleotide distributions are statistically different. The normality test failed only for the first class comprising $\mathrm{d}(\mathrm{GpG})$ dinucleotides. The other two sets passed both normality $(\mathrm{P}=0.134)$ and equal variance tests $(\mathrm{P}=0.096)$. I decided not including statistical analysis since the main goal of this paper was the answer to the question whether additivity is to be taken as important contribution to dinucleotide steps energetics. Thus, representative dinucleotide steps were taken into consideration and classified according to some common properties. Broad diversity of energy asymmetry coefficient was chosen as the selection criterion of dinucleotide steps. This ensured covering of the whole range of possible structures with minimized cost of computations. The presented quantification of non-additivities opens the door for more precise and broad characteristics of all possible di-, tri- and oligo- nucleotides. Since I have completed much more computations and I have now much extended point of view it seems to me that statistical analysis would be irrelevant in the context of this paper. Anyway I greatly appreciate this comment of the Referee.

4) Page 3, Method section

The MP2/avdz preoptimized geometries of monomers were used. I wonder what is the influence to the nonadditivy term if hydrogens were reoptimized within the base-pair? It would be worth evaluating this for some selected steps. I am asking this as in my experience there exist surprisingly not that small influence of hydrogen positions on the total stacking energies.

Thank you for this suggestion. This is really interesting subject, but I am afraid it is outside of the scope of this paper.

5) Page 3, Method section

$>$ From the Methods section it is not clear if the Cs symmetry was imposed during the monomer optimization. If yes, what is the influence to the nonadditivy term of keeping NH2 group, which is known to be pyramidalized, in plane?

I am aware that non-planarity of amino groups is a fact. However according to my experience this is quite small effect and I do not think it is crucial for anon-additivity. In fact full geometry optimization is required for proper evaluation of pyramidalization of $\mathrm{NH} 2$ exocyclic groups. Again although this aspect is interesting I must emphasize that it not the main focus of my interest but will surely be considered in forthcoming investigations.

6) Page 4

The point BD0015 (3-body term of $+24.2 \mathrm{kcal} / \mathrm{mol}$ and 4 -bod of $-21.2 \mathrm{kcal} / \mathrm{mol}$ ) is not present in the Figure 2. 
This is of course related to the applied scale. I have extended the sale for showing all points.

7) Page 4, end of the first paragraph

"The mean values of all ..." should be "The median values of all ...".

Thank you!

8) Page 4, end of the first paragraph

I don't think the following statement is true: "On the contrary for such steps as $d(A p A), d(T p A)$ and $\mathrm{d}(\mathrm{ApT})$ the nonadditivty has attractive nature." From Figure 2 it follows, that only AA is attractive, while AT and TA are repulsive. Similarly, the third class does not have all steps with repulsive nonadditivy (AA has attractive nonadditivity)

I have also updated Fig.3 and changed the text accordingly.

9) Concerning the third class, small value of nonadditivy term in steps with two AT pairs was already noticed e.g. in Ref 11, or recently in DOI: 10.1021/jp910788e. I think this should be acknowledged by proper reference.

Thank you for this prompt, of course I did it with pleasure.

10) I wonder whether the sign of the nonadditivity term doesn't depend on the level of theory used? Maybe if calculated at the very high level of theory (CCSD(T) complete basis set limit) instead MP2/avdz, the conclusions about the attractive/repulsive nature could be different. It i clear that such high level of theory is not doable, however some discussion would be appropriate.

I am afraid that at this moment the CCSD(T) level is beyond my computations capabilities for dinucleotide steps. Hence I only added warnings in the final section of my paper.

11) Also, MP2/avdz does not include dispersion non-additivity, this should be discussed (with appropriate references) in the text.

Thank you. Suitable comments were added. 


\title{
Non-additive interactions of nucleobases in model dinucleotide steps occurring in B-DNA crystals
}

Received: 08.12.2009 / Accepted: 12.04.2010

Piotr Cysewski ${ }^{1,2, \varpi}$

${ }^{1}$ Department of Physical Chemistry, Ludwik Rydygier Collegium Medicum in Bydgoszcz, Nicolaus Copernicus University in Toruń, Kurpińskiego 5, 85-950 Bydgoszcz, Poland, piotr.cysewski@cm.umk.pl

${ }^{2}$ Department of General Chemistry, University of Technology and Life Sciences in Bydgoszcz, ul. Seminaryjna 3, 85-326 Bydgoszcz, Poland

${ }^{\square}$ Email: piotr.cysewski@cm.umk.pl

\begin{abstract}
Non-additivity of base-base interactions in all ten possible model dinucleotide steps were analyzed on MP2/aug-cc-pvDZ quantum chemistry level. Conformations of four nucleobases exactly matched to ones occurring in B-DNA crystals. In most of 162 analyzed tetramers both three- and four-body contributions are negligible except of $\mathrm{d}(\mathrm{GpG})$ steps. However, in these dinucleotides both contributions are always of opposite signs and in all cases the sum of all non-additive part of intermolecular interactions do not exceed $2.6 \mathrm{kcal} \mathrm{mol}^{-1}$. This stands for less than $5 \%$ of the overall binding energy of dinucleotide steps. Also replacements of guanine with 8-oxoguanine in $\mathrm{d}(\mathrm{GpG})$ systems introduces non-additivity of the same magnitude as for canonical dinucleotides. It is observed linear relationships between values of total binding energy obtained in the tetramer basis set and estimated energy exclusively in dimers basis sets with assumption of pairwise additivities. For all analyzed dinucleotides steps there are also linear correlations between amount of non-additive contributions and magnitude of pairs interactions. Based on differences in electrostatic contribution to the total binding energy of four nucleobases and polarity of dinucleotide steps three distinct classes of dinucleotide steps were identified.
\end{abstract}

Keywords Many-body $\cdot$ Intermolecular interactions $\cdot$ Dinucleotide steps $\cdot 8$-oxoguanine B-DNA 


\section{Introduction}

Many-particle systems as immanent nature of physical world stand for emergence of new properties that cannot be derived just as simple adding up features of constituting elements. Non-negligible contributions of many-body terms to intermolecular interactions in molecular clusters are well known [1-4] and this aspect significantly complicates assessments of stabilization energies in multi-molecular systems. For example three-body terms have significant contribution in water clusters reaching even $30 \%$ to the interaction energy [2]. Fortunately, four-body and higher order terms are usually negligible [1-8]. This is of particular importance since almost all of molecular modeling procedures ignores non-additive contributions to the total energy of molecular systems. Typical example is the contemporary molecular dynamics simulations for biomolecules. Size of these kind of systems enable merely for application of force field parameterization inherently neglecting not only quantum effects but also all non-additivities of interacting systems. Implementing hybrid QM/MM methodology introduce improvement only to some extent. Furthermore, corrections for electron correlation are important contributions to amount of individual n-body terms frequently imposing increase of their magnitudes. On the other hand many-body contributions can substantially vary with the relative orientation of monomers $[5,6]$ and can originate from distinct types of interactions. For example in case of water molecules energies of hydrogen bonding are two order stronger than for van der Waals interactions in noble gas clusters. Although for electrically neutral and non-polar systems non-additivity is typically small [7] for polar systems or condensed phases non-additivity can become substantial [8]. A special example of system involving complicated interplay between many forces of different character [9-11] is found in the case of stabilization of polynucleotide chains. Many contributions are important for DNA involving contacts of its constituents as nucleobases, sugar moieties, phosphate backbones, surrounding water molecules, counter ions and diversity of ligands potentially present in solutions at physiological conditions. Even interactions between two heterocyclic nucleobases are non-homogeneous and typically are divided into three classes, namely hydrogen bonding, intra- and inter-strand stacking. It has been suggested that interaction between nucleic acids bases can in some extend exhibit such nonadditive nature [12-16]. Although base-base interactions in dinucleotide steps of B-DNA are usually pair-additive but in case of $\mathrm{d}(\mathrm{GpG})$ dinucleotide steps the absolute value of manybody contribution can be large and significantly structure-related [12-14]. As it was documented previously [12-16] high non-additive character of intermolecular interaction 
energy (IIE) has been found for many $\mathrm{d}(\mathrm{GpG})$ dinucleotide steps in B-DNA conformations. Although three- and four-body terms posses opposite signs they do not cancel each other and in most cases positive non-additivity is observed $[15,16]$. The origin of such unique properties of $\mathrm{d}(\mathrm{GpG})$ steps is expected [12-16] to come from electrostatic repulsion between polar guanine-cytosine pairs what also was related to polymorphic changes of guanine-rich sequences of DNA double helices [16-19]. On the other hand much weaker electrostatics occurring in $\mathrm{d}(\mathrm{ApT})$ steps do not introduces significant non-additivity. However, small values of non-additive terms were also noticed [13, 14] for steps comprising two AT pairs. Many molecular clusters comprising for example water, ammonia or formamide molecules [2-4] exhibit similar sensitivity to polarization effects. Unfortunately, the level of theory used for characteristics of intermolecular interactions is a crucial factor determining magnitude of binding energies. This is related not only to level of electron correlation estimation [11-19], corrections of basis superposition errors (BSSE) [20] and quality of applied basis set [11-14] (or its extrapolation to complete one) but also to non-additive contributions to molecular cluster stabilizations [12-16]. As it was nowadays documented [16] accurate values for manybody effects require extensions of electron correlation beyond MP2 levels since this approximation can only encounter non-additivity via correlated exchange and induction components [21, 22]. However, due to fortunate compensation of errors such levels of theory as MP3, L-CCD or CCSD lead to substantially the same predictions as CCSD(T) one [16]. Due to the computer resources required for characteristics of nucleobases clusters there are only few papers quantifying the non-additive nature of intermolecular interactions [13-15, 2325]. Unfortunately the size of analyzed systems prohibit application of advanced levels of theory as routine computations for broad range of nucleobases conformations. Bearing in mind formal restrictions of MP2 level [21, 22] the qualitative estimation of non-additive nature of dinucleotide steps was characterized here for all ten unique dinucleotide steps adopting B-DNA conformations. Let consider schematic representation of model dinucleotide step as it is presented in Fig. 1. The total binding of four nucleobases in such system can be written in the following general form:

$$
\Delta E_{d(X p Y)}^{\text {total }}=E_{d(X p Y)}^{\text {tetr }}-\sum_{i=1}^{4} E_{i}^{\text {tetr }}
$$

The 5'-X and 3'-Y monomers present in the strand $\mathrm{I}$ are numbered as 1 or 2 , whereas corresponding complementary bases placed in the strand II are labeled as 4 or 3, respectively. From the formal point of view all terms are to be estimated in the basis sets of the whole 
tetramer, what was indicated by tetr superscript. The decomposition of the total interaction energy into clusters of multi-body expansion can be defined as follows [26, 27]:

$$
\Delta E_{d(X P Y)}^{\text {total }}=\sum_{j>i=1}^{4} \varepsilon_{i j}^{(2)}+\sum_{k>j>i=1}^{4} \varepsilon_{i j k}^{(3)}+\varepsilon_{d(X p Y)}^{(4)}
$$

The analyzed dinucleotide steps are characterized by the following six two-body terms describing pairwise interactions between monomers:

$$
\varepsilon_{i j}^{(2)}=E_{i j}^{\text {tetr }}-E_{i}^{\text {tetr }}-E_{j}^{\text {tetr }} \quad\{\mathrm{i}, \mathrm{j}>\mathrm{i}\}=\{1,2,3,4\}
$$

The subscripts denote monomers according to notation used in Fig. 1. For the dinucleotide steps there are four tree-body terms defined as follows:

$$
\varepsilon_{i j k}^{(3)}=E_{i j k}^{\text {tetr }}-E_{i}^{\text {tetr }}-E_{j}^{\text {tetr }}-E_{k}^{\text {tetr }}-\varepsilon_{i j}^{(2)}-\varepsilon_{i k}^{(2)}-\varepsilon_{j k}^{(2)} \quad\{\mathrm{i}, \mathrm{j}>\mathrm{i}, \mathrm{k}>\mathrm{j}\}=\{1,2,3,4\}
$$

Finally, the only four body term adopts one straightforward form:

$$
\varepsilon_{d(X p Y)}^{(4)}=E_{d(X p Y)}^{t e t r}-\sum_{i=1}^{4} E_{i}^{\text {tetr }}-\sum_{j>i=1}^{4} \varepsilon_{i j}^{(2)}-\sum_{k>j>i=1}^{4} \varepsilon_{i j k}^{(3)}
$$

The additive part of the intermolecular interaction energy is usually defined as the sum of all two-body terms but all higher order components constitute so-called non-additive part. The above scheme, although very simple and straightforward, is rather time-consuming and usually not-applicable for large number of dinucleotide conformations and advanced quantum chemistry levels. Thus, the most obvious simplification is neglecting of all non-additive terms and estimation of all energies exclusively in the basis sets of dimers:

$$
\Delta E_{d(X p Y)}^{a d d, \operatorname{dim}}=\sum_{j>i=1}^{4}\left(E_{i j}^{d i m}-E_{i}^{d i m}-E_{j}^{d i m}\right)
$$

In such procedure monomers energies are recomputed separately for each pair but the overall CPU cost is about one order lower than full computations in tetramer basis set. The following difference quantifies the error of pairwise additivity of binding energy:

$$
\Delta E_{d(X p Y)}^{\text {non-add }}=\Delta E_{d(X p Y)}^{\text {total }}-\Delta E_{d(X p Y)}^{\text {add, dim }}
$$


The aim of this study is the quantitative analysis of magnitude of non-additive contributions to the total binding energy of dinucleotide steps and validation of assumption of pair-wise additivities of dimers stabilization energies. Besides, non-standard dinucleotide steps were considered that comprise one 8-oxoguanine molecule denoted by $\mathrm{Z}$ in two alternative sequences $\mathrm{d}(\mathrm{ZpG})$ or $\mathrm{d}(\mathrm{GpZ})$.

\section{Methods}

Model $\mathrm{d}(\mathrm{XpY})$ steps were prepared according to procedure applied in our previous investigations [28-32] used for description of energetic heterogeneities in canonical and oxidized central guanine triad of B-DNA telomeric fragments [28, 29], polymorphism-related heterogeneities of guanine stacking in B- and A-DNA forms [30], characteristics of inter- and intra-strand stacking interactions in $\mathrm{d}(\mathrm{CpG})$ and $\mathrm{d}(\mathrm{GpC})$ steps found in B-DNA, A-DNA and Z-DNA crystals [31], quantification of all possible intra-strand stacking interactions between nucleobases [32] and assessment of many-body contributions to $\mathrm{d}(\mathrm{GpG})$ and $\mathrm{d}(\mathrm{CpC})$ dinucleotide steps [15]. The essential feature of applied approach is the characteristics of statistically significant number of conformations leading to distributions of intermolecular interaction energies (IIE) along with standard deviations and other statistical parameters. According to Standard Reference Frame [33] the complete description of three-dimensional arrangements of bases in nucleic acid structures is achieved by definition of values of base pair (shear, stretch, stagger, buckle, propeller and opening) and base pair step (shift, slide, rise, tilt, roll and twist) parameters. The values of these parameters corresponding to B-DNA crystals were taken from the Nucleic Acid Database [34] for all dinucleotide steps comprising canonical nucleobases. Preparation of model dinucleotide steps was done with an aid of X3DNA program [35], in which fragments library was modified by replacement of native geometries (without hydrogen atoms) with monomers pre-optimized on MP2/aug-cc-pvDZ (aDZ) level (imposing $\mathrm{C}_{\mathrm{s}}$ symmetry). These monomers along with sets of 18 parameters (six for either hydrogen bonded pair and additional six for stacked two-bases pairs) were used for preparation of tetramers in which orientations of nucleobases exactly correspond to ones found in the original B-DNA structures. In all cases the whole sugar-phosphate backbones were simplified just by hydrogen atoms. All ten unique model $\mathrm{d}(\mathrm{XpY})$ dinucleotide steps were used for single point energy estimation using density fitting MP2/aug-cc-pVDZ (aDZ) level of theory. The counterpoise correction for BSSE error [20] was included in all single 
point calculations of dimers, trimers and tetramers. The MolPro package [36] was used in quantum chemistry calculations. Many body contributions were characterized according to formulas presented in the proceeding section.

\section{Results and discussion}

According to procedure described above there were analyzed 162 model dinucleotide steps including 48 of $\mathrm{d}(\mathrm{GpG}), 23$ of $\mathrm{d}(\mathrm{ApA}), 16$ of $\mathrm{d}(\mathrm{CpG}), 15$ of $\mathrm{d}(\mathrm{TpA}), 10$ of either $\mathrm{d}(\mathrm{GpC})$, $\mathrm{d}(\mathrm{GpA}), \mathrm{d}(\mathrm{ApG}), \mathrm{d}(\mathrm{ApC}), \mathrm{d}(\mathrm{CpA})$ and $\mathrm{d}(\mathrm{ApT})$. All analyzed dinucleotide steps were described in Table S1 in supplementary material. As it was schematically presented in Fig. 1 there are three distinct types of intermolecular interactions that stabilize model dinucleotide steps. The most dominant contribution to the total binding comes from two-body interactions typically described as hydrogen bonding, inter- and intra-strand stacking. Three-body interactions consist of four distinct terms involving all combinations of intra-strand stacked pair interacting with one nucleobase via hydrogen bonding. Finally, there is only one fourbody term.

\section{The magnitude of many body contributions}

The calculated values of non-additive contributions to intermolecular interaction energy in ten possible model dinucleotide steps are presented in Fig. 2 and 3. First of all it is visible distinct nature of $\mathrm{d}(\mathrm{GpG})$ steps since in most cases significant non-additive part is obtained. For this step the distributions of both three- and four-body terms can vary significantly as it is presented in Fig. 2. The strongest attraction coming from three-body terms has been found for $\mathrm{d}(\mathrm{GpG})$ dinucleotide step in B-DNA coded as BD0085 and it is as high as $-18.9 \mathrm{kcal} \mathrm{mol}^{-1}$. The corresponding value of four-body term is also unusually high and equals $+20.2 \mathrm{kcal} \mathrm{mol}^{-}$

1 . On the contrary in another utmost case (BD0015) it is noticed extremely high repulsion coming from three-body term $\left(+24.2 \mathrm{kcal} \mathrm{mol}^{-1}\right)$ and associated attraction originating from four-body term $\left(-21.2 \mathrm{kcal} \mathrm{mol}^{-1}\right)$. However, these two examples are rare extremes since more than $90 \%$ of analyzed $\mathrm{d}(\mathrm{GpG})$ steps are characterized by three-body terms lower than $+6 \mathrm{kcal}$ $\mathrm{mol}^{-1}$. The corresponding span of four-body contributions is from $-5 \mathrm{kcal} \mathrm{mol}^{-1}$ to $+2 \mathrm{kcal}$ $\mathrm{mol}^{-1}$. Closer inspection [15] reveals that not all three-body contributions are of the same magnitude for $\mathrm{d}(\mathrm{GpG})$ steps. All terms, except 134 one are usually quite small and typically 
do not exceed $2.0 \mathrm{kcal} \mathrm{mol}^{-1}$. However, interactions stabilizing 134 trimer have completely different nature. These contributions can have magnitude of several $\mathrm{kcal} \mathrm{mol}^{-1}$ and are the main source of non-additivity in $\mathrm{d}(\mathrm{GpG})$ steps. This three-body cluster involves stacking of two cytosine molecules in strand II and hydrogen bonding of 5'-guanine present in strand I. Interestingly, although three-body contributions may have attractive or repulsive character, always opposite sign of four-body term is observed. However, this does not lead to cancelation of many body contributions and in case of $\mathrm{d}(\mathrm{GpG})$ step the total non-additivity is repulsive. As it was presented in Fig. 2 many-body contributions found for all other dinucleotide steps are much smaller and can be assumed as negligible since three-body terms are always within $-1.8 \div 1.7 \mathrm{kcal} \mathrm{mol}^{-1}$ and four-body ones belong to interval $-0.7 \div 0.2 \mathrm{kcal}$ $\mathrm{mol}^{-1}$. Since stabilization energy of model dinucleotide steps usually exceed $-40 \mathrm{kcal} \mathrm{mol}^{-1}$ such small non-additivity affects at most modestly the overall biding of four nucleobases in $\mathrm{d}(\mathrm{XpY})$ steps. The median values of all non-additive contributions were presented in Fig. 3. Although all medians are positive the dinucleotide steps can be divided into three classes. As it was mentioned above the $\mathrm{d}(\mathrm{GpG})$ steps are characterized by highest repulsion coming from non-additive interactions. On the contrary for $\mathrm{d}(\mathrm{ApA}), \mathrm{d}(\mathrm{TpA})$ and $\mathrm{d}(\mathrm{ApT})$ dinucleotides nonadditivity although positive is close to zero. The third class comprises the rest of dinucleotide steps, for which non-additivity is quite modest and repulsive. The statistical analysis applied to these three classes of dinucleotide steps proved that the differences in the median values are greater than would be expected by chance $(\mathrm{P}=<0.001)$. Hence all three dinucleotide distributions are statistically different. The normality test failed only for the first class comprising $\mathrm{d}(\mathrm{GpG})$ dinucleotides. The other two sets passed both normality $(\mathrm{P}=0.134)$ and equal variance tests $(\mathrm{P}=0.096)$.

\section{Linear relationships of intermolecular interactions}

Since the intermolecular interactions between canonical and modified nucleobases are important aspect characterizing polynucleotide chains there is great temptation in assuming pairwise additivity of interactions stabilizing tetramers. This is justified not only by economy of computations but also by results presented in the previous section. However, in some cases, as for example for extreme conformations of $\mathrm{d}(\mathrm{GpG})$ steps, non-additive contributions can be significant. Thus, it is interesting to analyze the relationship between exact values of dinucleotide stabilization energies and simplified ones obtained after neglecting of all non- 
additive terms in the binding energy of $\mathrm{d}(\mathrm{XpY})$ steps and utilization of exclusively dimer basis sets. Thus, the following formula is proposed for regression analysis:

$$
\Delta E_{d(X p Y)}^{\text {total }, \text { st }}=a \cdot \Delta E_{d(X p Y)}^{a d d, \operatorname{dim}}+b
$$

where $\mathrm{a}$ and $\mathrm{b}$ are regression coefficients and left-hand side estimates the total binding of nucleobases in $\mathrm{d}(\mathrm{XpY})$ step as the best fitted values to exact IIE of sum of pairwise additive terms estimated in dimer basis set. The applied regression analysis leads to plots provided in Fig. 4. As one could expect there is observed quite acceptable linear correlation between total binding energy and one estimated as simple sum of pairs energies (in the basis set of dimers). Even extreme cases for which dinucleotide steps are energetically unfavorable $(+3.28 \mathrm{kcal}$ $\mathrm{mol}^{-1}$ in case of $\mathrm{d}(\mathrm{ApC})$ found in BDL035 or with very small attraction $-6.1 \mathrm{kcal} \mathrm{mol}^{-1}$ for d(ApA) (present in BDJ081) fulfill linear-dependence. At first glance one could recommend utilization of only one linear regression function in Fig. 4, but previously analyzed mean values of all non additive terms (see Fig. 3) suggested that three different classes of dinucleotide steps are to be considered. Since regressions presented in Fig. 4 allow for estimation of not only the total binding but also non-additive part it is interesting to see if linear relationships also hold for the latter quantity. Interestingly, due to different origin of non-additive parts in dinucleotide steps application of only one regression line would lead to very poor estimation of non-additive terms. On the contrary, as it was presented in Fig. 5, three relationships lead to quite acceptable description of non-additive terms by exclusively pairs interactions. Hence, there are three classes of dinucleotide steps with respect of manybody contributions to the total binding energy in dinucleotide steps. This can be further justified by values presented in Table 1, which collects the mean values of SCF and electron correlation contributions to the total values of IIE. Besides, the mean values of dipole moments of analyzed dinucleotide steps are also provided. Interestingly, although all SCF energies are negative indicating strong electrostatic contribution to the total binding energy, there are observed quite significant diversities between particular dinucleotide steps. First of all $\mathrm{d}(\mathrm{ApA}), \mathrm{d}(\mathrm{ApT})$ and $\mathrm{d}(\mathrm{TpA})$ systems are characterized by highest values of SCF term. Additionally, these tetramers belong to the less polar steps. On the contrary the $d(G p G)$ is known $[11,12]$ as the most polar system among all ten possible dinucleotide steps, what also is confirmed by mean values of dipole moment collected in Table 1. Besides, much lower values of SCF contribution are noticed in this case if compared to previously described set of dinucleotides. The rest tetramers constituting class of dinucleotide steps that is characterized by much stronger attraction coming from electron correlation energy and are of modest 
polarity. Thus, polarity expressed in terms of dipole moments along with electron correlation and SCF contribution to the total binding energy are quite satisfactory criterions of classification of dinucleotide steps into three distinct classes. Although the absolute values of non-additive terms seem to be negligible they are still interesting source of distinguishing of dinucleotide steps.

\section{Additivity of interactions in dinucleotide steps comprising 8-oxoguanine}

Many products of nucleobases oxidation were identified in numerous irradiation and chemical experiments both in vivo and in vitro conditions of DNA and DNA-protein complexes (chromatin) [37, 38]. The use of gas chromatography-mass spectrometry with selected-ionmonitoring technique proved steady state levels of many free-radical derived analogues of canonical nucleobases [39]. They are believed to play crucial role in ageing and different stages of cancer development [37, 38]. The structural and energetic consequences of B-DNA oxidation were not described till now. It is reasonable to expect that presence of 8-oxoguanine in B-DNA can significantly alter intermolecular interactions [40]. For example intra-strand stacking interactions of $8-\mathrm{oxoG}$ with guanine as well as hydrogen bonding with cytosine are stronger compared to canonical guanine at least in telomere repeat units [28, 29]. It is interesting to see if modification of guanine leads also to any non-additivities in base-base interactions occurring in oxidized dinucleotide steps. Since there are not available sufficient amount of experimental data comprising 8-oxoguanine alternatively molecular dynamics simulations were used for collecting structural parameters defining oxidized dinucleotide steps. Details are provided elsewhere [40]. The decomposition of dinucleotide stabilizing energies into many-body contributions can be found in Fig. 6. Presence of 8-oxoguanine in $\mathrm{d}(\mathrm{ZpG})$ or $\mathrm{d}(\mathrm{GpZ})$ dinucleotide steps introduces significant changes upon non-additivity. First of all, unlike to canonical $\mathrm{d}(\mathrm{GpG})$ sequence, both three- and four-body contributions are usually of the same sign. Thus, although actual values of non-additive terms are smaller if compared to canonical guanine dinucleotides it is expected that cumulative effect occurs. The mean values of all three-body terms is about +2.0 and $+2.5 \mathrm{kcal} \mathrm{mol}^{-1}$ for $\mathrm{d}(\mathrm{ZpG})$ and $\mathrm{d}(\mathrm{GpZ})$ dinucleotide steps, respectively. The mean values of four body contributions are much smaller and do not exceed $0.13 \mathrm{kcal} \mathrm{mol}^{-1}$ in either case. Thus, many-body contributions are of repulsive character reducing total stabilization of the two stacked pairs. The mean values of non-additivities characterizing oxidized dinucleotide steps can reach $5 \%$ of total IIE. This is similar amount to one found in case of $d(\mathrm{GpG})$ steps [15]. No sequence dependence of many 
body contributions is observed for oxidized dinucleotides. There is another interesting features of three body terms found for modified dinucleotide steps. Majority of 123 and 134 contributions are systematically smaller than 124 and 234 ones. The common patterns in these terms are formed by purine-pyrimidine inter-strand stacking $\left(\mathrm{G}_{1}\left|\mathrm{C}_{3}, \mathrm{Z}_{1}\right| \mathrm{C}_{3}, \mathrm{C}_{2} \mid \mathrm{G}_{4}\right.$ or $\left.\mathrm{C}_{2} \mid \mathrm{Z}_{4}\right)$. It seems that 13 interactions introduces attractions in analyzed steps, while 24 inter-strand interactions are responsible for repulsive contributions irrespectively of the analyzed sequences. In case of $\mathrm{d}(\mathrm{GpG})$ this feature was not observed [15] since major contributions to the total non-additivity came from 134 term. Finally, it is worth mentioning that there are linear relationships between full estimation of IIE values and ones utilizing pair-wise additive assumption. The applied linear regression analysis led to quite acceptable correlation with $\mathrm{R}^{2}=0.983$ for oxidized dinucleotide steps and $\mathrm{R}^{2}=0.996$ for canonical ones. This relationship is presented in subsequent study of this issue [40]. Finally, the total binding of four nucleobases in oxidized $\mathrm{d}(\mathrm{GpG})$ steps is about $2 \mathrm{kcal} \mathrm{mol}^{-1}$ stronger than found for canonical dinucleotide.

\section{Conclusions}

Results presented above qualitatively characterize non-additivity of base-base interactions stabilizing $\mathrm{d}(\mathrm{XpY})$ dinucleotide steps. Although applied model excluded sugar-phosphate backbone, the most important features of intra-and inter-strand stacking along with hydrogen bonding were properly preserved. The most important aspects of presented data is rational representation of four bases conformations and adequate level of computations. The first facet relies on the representation of meaningful conformations matching to ones occurring in BDNA crystals. The main conclusion drawn from presented results is that pair-wise additivity simplification is rational since corresponding intermolecular interaction energy of $\mathrm{d}(\mathrm{XpY})$ steps suffers far less than $5 \%$ if compared to correct values. The four- and three- body terms are small and usually of the opposite signs, what further reduces its impact on the total binding energy. Dinucleotide steps comprising two stacked guanine molecules seem to be the only canonical systems for which non-additivity is non-negligible. However, as it was demonstrated in the previous report [15], in this case there are observed linear relationships which allow for corrections of IIE values without necessity of energy computations for the whole tetramer. Despite the fact that many body contributions are small, they can be found as interesting source of classification of dinucleotide steps into three distinct classes. First class 
comprised $\mathrm{d}(\mathrm{GpG})$ steps that were found as most polar among all dinucleotide steps. To the second class one can include $\mathrm{d}(\mathrm{ApA}), \mathrm{d}(\mathrm{TpA}$ and $\mathrm{d}(\mathrm{ApT})$ steps. They are characterized by highest values of SCF contributions to the total IIE and lowest polarities. The rest of dinucleotide steps constitute the third class with modest polarity and electrostatic contribution to the total interaction energy. Oxidation of guanine in $\mathrm{d}(\mathrm{GpG})$ dinucleotide steps introduces non-negligible non-additivity to IIE that is of the same order as observed for canonical systems. In the case of many potential structures contribution to analyzed quantity there is always question related to arbitral selection of analyzed systems. It is impractical to analyze hundreds of $\mathrm{d}(\mathrm{XpY})$ conformations and misfortune sampling may significantly affect conclusions. However, in the previous paper [15] it was demonstrated that there is simple origin of non-additivity in the case of $\mathrm{d}(\mathrm{GpG})$ dinucleotide steps. The interactions between hydrogen bonded nucleobases as well as inter-strand stacking interactions can vary significantly for different conformations of four bases. As the quantitative measure of such feature the energy asymmetry coefficient $(\delta)$ is proposed. It is defined as the sum of two following terms. First one denotes the difference in hydrogen bonding energies between two neighboring complementary nucleobases and the second one characterizes difference between 5-G $\mid \mathrm{C}^{\prime}-5^{\prime}$ and 3-G|C'-3' inter-strand stacking interactions. In case of $\mathrm{d}(\mathrm{GpG})$ dinucletode steps this parameters emphasizes the importance of hetero-bases contacts. There is straightforward way of extension of $\delta$ coefficient for other tetramers and characterize the symmetry/asymmetry diversities (in energy sense) of all dinucleotide steps. In any case close to zero values of $\delta$ coefficient indicate high symmetry of intermolecular interactions. On the contrary the positive values of this parameter suggest stronger interactions on 3' side and vice versa negative values emphasizes stronger attraction on $5^{\text {' }}$-side. In Fig. 7 there is presented distribution of $\delta$ values estimated for analyzed dinucleotide steps. As is it visible all analyzed systems may adopt wide range of energetic asymmetry covering broad range of structures. Hence, despite analogical distributions of energetic asymmetry the high non-additive contributions are observed only for canonical and oxidized $d(G p G)$ steps. This suggest that the selection of dinucleotide conformations is adequate and presented conclusions are expected to be consistent also for other conformations of dinucleotide steps. The results presented in this paper are preliminary and further investigations are necessary. Several aspects were excluded from the analysis that probably can affect both stabilization energies of dinucleotide steps and many-body contributions. Among them the applied level of computations is the first way of improvements. More sophisticated methods should be applied for proper inclusion of electron correlations. Although, presented results embrace only small 
portion of this effect due to applied level of computations it is reasonable to expect that extension of the method will not change the general conclusion of the magnitude of manybody contributions do dinucleotide steps energetic. Besides there are other aspect worth additional considerations. For example it is commonly known that nucleobases comprising amino groups are not planar. These exocyclic substituent exhibit pyramidalization, which probably affects both IIE and many-body terms. Also relaxation of monomers geometry, especially hydrogen bonds are worth of precise evaluation. The importance of all these aspects is to be validated in further investigations.

\section{Acknowledgments}

Results were obtained the part of computational grant no 39 of Poznan Supercomputing and Networking Center (PSNC, Poznań, Poland). The allocation of computational facilities are greatly appreciated. 


\section{References}

1. Elrod MJ, Saykally RJ (1994) Chem Rev 94:1975-1997

2. Xantheas SS (1994) J Chem Phys 100:7523-7534

3. Suhai SJ (1994) Chem Phys 103:7030-7039

4. Saykally R (1996) Science 271:929-933

5. Habitz P, Bagus, Siegbabn P, Clementi E (1983) Int J Quantum Chem. 23:1803-1806

6. Chalasinski G, Szczesniak MM, Cieplak P, Sheiner S (1991) J Chem Phys 94:2873-2883

7. Bukowski R, Szalewicz K (2001) J Chem Phys 114:9518-9531

8. Bukowski R, Szalewicz K, Groenenboom GC, van der Avoird A (2008) J Chem Phys 128:094314

9. Saenger W (1998) Principles of Nucleic Acid Structures. Springer, Berlin

10. Bloomfield VA, Crothers DM, Tinoco I (1999) Nucleic Acids: Structures, Properties and Functions. University Science Books, Sausalito, CA

11. Hobza P, Šponer J (1999) Chem Rev 99:3247-3276

12. S Śponer J, Riley KE, Hobza P (2008) Phys Chem Chem Phys 10:2595-2610

13. Šponer J, Gabb HA, Leszczynski J, Hobza P (1997) Biophys J 73:76-87

14. Pitoňák, P. Neogrády, Hobza P (2010) Phys Chem Chem Phys 12:1369-1378

15. Cysewski P (2010) Int J Quantum Chem DOI:10.1002/qua.22435

16. Svozil D, Hobza P, Šponer J (2010) J Phys Chem B 114:1191-1203

17. Lankas F, Cheatham TE, Špaĉkova N, Hobza P, Langowski J, Šponer J (2002) Biophys J 82:2592-2609

18. Ng HL, Dickerson RE (2002) Nucleic Acids Res 30:4061-4067

19. Šponer J, Florian J, Ng HL, Šponer J, Špaĉkova N (2000) Nucleic Acids Res 24:48934902

20. Boy SF, Bernardi F (1970) Mol Phys 19:553-556

21. Hesselmann A, (2008) J Chem Phys 128:144112

22. Chalasinski G, Szczesniak MM (1994) Chem Rev 94:1723-1765

23 Peters M, Rozas I, Alkorta I, Elguero J (2003) J Phys Chem B 107:323-330

24. Gu J, Wang J, Leszczynski J (2004) J Phys Chem B 108:8017-8022

25. Biot C, Wintjens R, Rooman M (2004) J Am Chem Soc 126:6220-6221

26. Hankins D, Moskowitz JW, Stillinger FH (1979) J Chem Phys 53:4544-4554

27. Mierzwicki K, Latajka Z (2000) Chem Phys Letters 325:465-472

28. Cysewski P, Czeleń P (2007) J Mol Model 13:739-750 
29. Cysewski P, Czeleń P (2009) J Mol Mod 15:607-613

30. Cysewski P (2008) J Mol Struct THEOCHEM 865:36-43

31. Cysewski P (2009) J Mol Mod 15:597-606

32. Cysewski P (2009) New J Chem 33:1909-1917

33. Olson WK, Bansal M, Burley SK, Dickerson RE, Gerstein M, Harvey SC, Heinemann U, Lu XJ, Neidle S, Shakked Z, Sklenar H, Suzuki M, Tung CS, Westhof E, Wolberger C, Berman HM (2001) J Mol Biol 313(1):229-237

34. Berman HM, Olson WK, Beveridge DL, Westbrook J, Gelbin A, Demeny T, Hsieh S-H, Srinivasan AR, Schneider B (1992) Biophys J 63:751-759

35. Lu XJ, Olson WK (2003) Nucleic Acids Res 31:5108-5121

36. Werner HJ, Knowles PJ, Lindh R, Manby FR, Schutz M, Celani P, Korona T, Rauhut G, Amos RD, Bernhardsson A, Berning A, Cooper DL, Deegan MJO, Dobbyn AJ, Eckert F, Hampel C, Hetzer G, Lloyd AW, McNicholas SJ, Meyer W, Mura ME, Nicklaß A, Palmieri P, Pitzer R, Schumann U, Stoll H, Stone AJ, Tarroni R and Thorsteinsson T, MOLPRO, a package of ab initio programs designed by Werner HJand Knowles PJ, (2006) Version 2006.0 (Patch 2006.1) Cardiff, UK

37. von Sonntag C (2006) Free-Radical-Induced DNA Damage and Its Repair, A Chemical Perspective. Springer-Verlag, Berlin, Heidelberg, New York

38. Rice-Evans C, Halliwell B, Lunt GG (1995) Free Radicals and Oxidative Stress : Environment, Drugs and Food Additives, Portland Press, London

39. Halliwell B, Gutteridge JMC (1999) Free Radicals in Biology and Medicine, Clarendon Press, Oxford 1999

40. Cysewski P, Czeleń P (2010) J Mol Mod submitted to this issue (under reviewing process) 


\section{Tables}

Table 1 The mean values of dipole moments, SCF and electron correlation contributions to the total binding energy of model dinucleotide steps. Energies are expressed in $\mathrm{kcal} \mathrm{mol}^{-1}$ and dipole moment are given in Debyes. Bold face was used for demonstration of differences between three distinct classes of dinucleotide steps

\begin{tabular}{|c|c|c|c|c|}
\hline class & step & $\overline{\Delta E}_{d\left(X_{p} Y\right)}^{\text {total } S C F}$ & $\overline{\Delta E}_{d(X p Y)}^{\text {total,correl }}$ & $\begin{array}{c}\text { dipole } \\
\text { moment }\end{array}$ \\
\hline I & $\mathrm{d}(\mathrm{GpG})$ & $-16.9 \pm 13.1$ & $-51.0 \pm 10.7$ & $\mathbf{1 1 . 8} \pm \mathbf{1 . 3}$ \\
\hline II & $\mathrm{d}(\mathrm{CpG})$ & $-24.7 \pm 16.0$ & $\mathbf{- 5 6 . 7} \pm \mathbf{1 3 . 6}$ & $2.7 \pm 1.3$ \\
\hline & $\mathrm{d}(\mathrm{GpC})$ & $-29.2 \pm 1.9$ & $\mathbf{- 6 4 . 9} \pm \mathbf{1 . 3}$ & $4.8 \pm 0.4$ \\
\hline & $\mathrm{d}(\mathrm{GpA})$ & $-13.2 \pm 6.9$ & $\mathbf{- 4 7 . 7} \pm \mathbf{4 . 7}$ & $7.2 \pm 0.3$ \\
\hline & $\mathrm{d}(\mathrm{ApG})$ & $-11.3 \pm 13.5$ & $\mathbf{- 4 4 . 2} \pm \mathbf{1 2 . 0}$ & $8.4 \pm 0.3$ \\
\hline & $\mathrm{d}(\mathrm{ApC})$ & $-6.2 \pm 23.5$ & $\mathbf{- 4 4 . 2} \pm \mathbf{1 6 . 8}$ & $4.5 \pm 0.5$ \\
\hline & $\mathrm{d}(\mathrm{CpA})$ & $-23.3 \pm 2.2$ & $\mathbf{- 5 2 . 0} \pm \mathbf{0 . 4}$ & $4.5 \pm 0.3$ \\
\hline III & $\mathrm{d}(\mathrm{ApA})$ & $\mathbf{- 2 . 2} \pm \mathbf{6 . 3}$ & $-38.3 \pm 4.7$ & $3.9 \pm 0.4$ \\
\hline & $\mathrm{d}(\mathrm{ApT})$ & $\mathbf{- 0 . 9} \pm \mathbf{4 . 7}$ & $-37.6 \pm 5.6$ & $1.6 \pm 1.3$ \\
\hline & $\mathrm{d}(\mathrm{TpA})$ & $\mathbf{0 . 3} \pm \mathbf{1 2 . 2}$ & $-32.3 \pm 8.4$ & $1.7 \pm 0.4$ \\
\hline
\end{tabular}




\section{Figure captions}

Fig. 1 The schematic representation of intermolecular interactions in model $d(X p Y)$ dinucleotide steps. There are three distinct types of contacts, namely intra-strand stacking $\left(5^{\prime}-\mathrm{X}_{1} / \mathrm{Y}_{2}-3^{\prime}, 5^{\prime}-\mathrm{Y}^{\prime}{ }_{3} / \mathrm{X}^{\prime}{ }_{4-3} 3^{\prime}\right)$, inter-strand stacking $\left(5^{\prime}-\mathrm{X}_{1} \mid \mathrm{Y}^{\prime}{ }_{3}-5^{\prime}, 3^{\prime}-\right.$ $\left.\mathrm{Y}_{2} \mid \mathrm{X}^{\prime}{ }_{4} 3^{\prime}\right)$ and hydrogen bonding $\left(5^{\prime}-\mathrm{X}_{1}-\mathrm{X}_{4^{-}} 3^{\prime}, 3^{\prime}-\mathrm{Y}_{2}-\mathrm{Y}^{\prime}{ }_{3} 5^{\prime}\right)$, where $\mathrm{X}, \mathrm{Y}$ denotes one of four nucleobases and $X^{\prime}, Y^{\prime}$ stands for corresponding complementary base

Fig. 2 The values of non-additive contributions to IIE energy of all ten possible model $d(X p Y)$ dinucleotide steps, where $\{(X, Y\}=\{A, G, C, T\}$. Black circles denote sum of all three-body contributions, gray circles correspond to four-body term and open triangles represent the sum of all non-additivities

Fig. 3 The median values of all non additive terms estimated for ten possible dinucleotide steps

Fig. 4 The correlation between full estimation of total intermolecular interaction energies in dinucleotide steps including all non-additive terms in tetramer basis set $\left(\right.$ total $\left.\Delta \mathrm{E}=\Delta E_{d\left(X_{X Y}\right)}^{\text {total }}=E_{d\left(X_{P} Y\right)}^{\text {tetr }}-\sum_{i=1}^{4} E_{i}^{\text {tetr }}\right)$ and simplified assessment obtained after assumption of pairwise additivity of base-base interactions in dimer basis sets $\left(\right.$ additive $\Delta \mathrm{E}=\Delta E_{d\left(X_{p} Y\right)}^{\text {add dim }}=\sum_{j>i=1}^{4}\left(E_{i j}^{\text {dim }}-E_{i}^{d i m}-E_{j}^{d i m}\right)$

Fig. 5 The applicability of linear relationships obtained in Fig. 4 for estimation of nonadditive contributions based on pair interactions estimated using dimer basis sets

Fig. 6 Distribution of many body contributions to intermolecular interaction in oxidized $\mathrm{d}(\mathrm{GpG})$ dinucleotide steps. Open circles denote $\mathrm{d}(\mathrm{GpZ})$ steps, while black circles stand for $\mathrm{d}(\mathrm{ZpG})$ sequence, where $\mathrm{Z}$ denotes 8-oxoguanine

Fig. 7 The distribution of energy asymmetry coefficient $(\delta)$ estimated for all of analyzed dinucleotide steps 


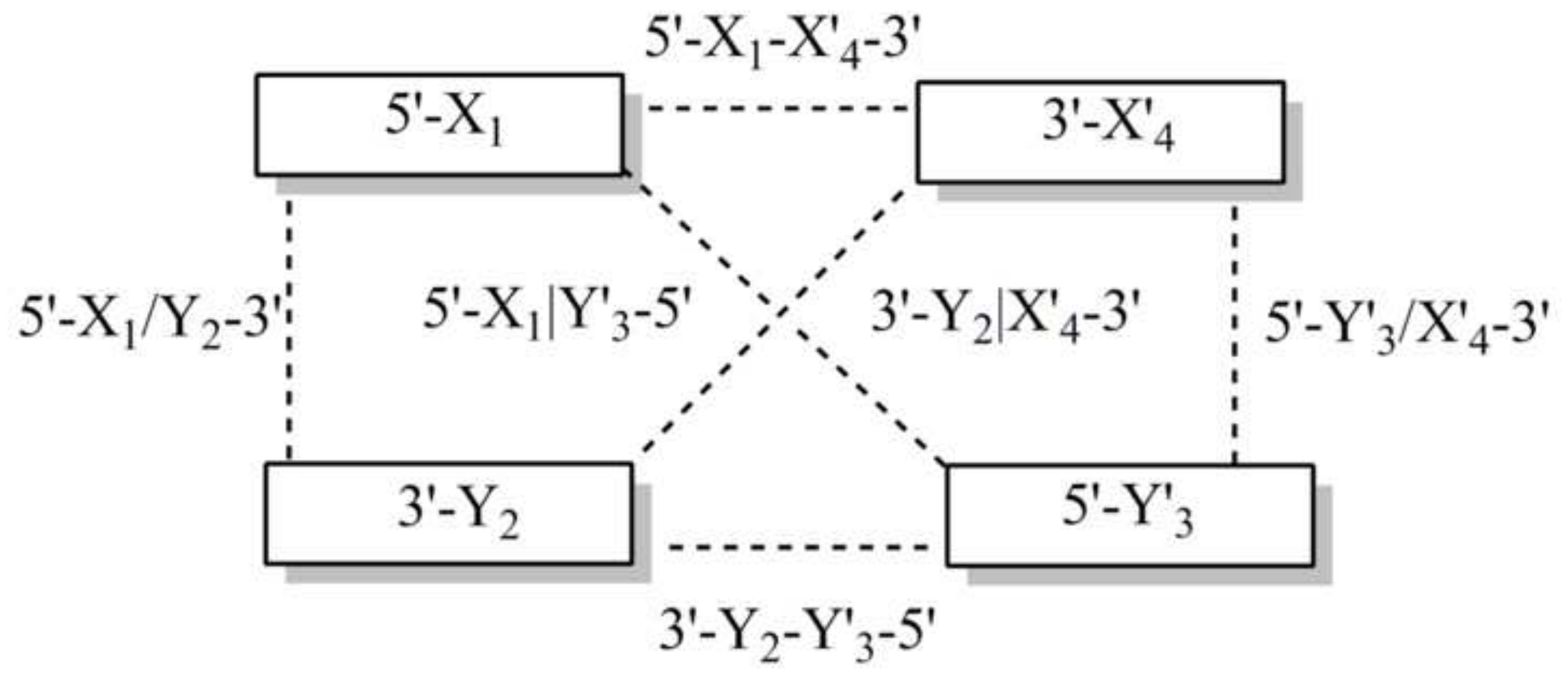




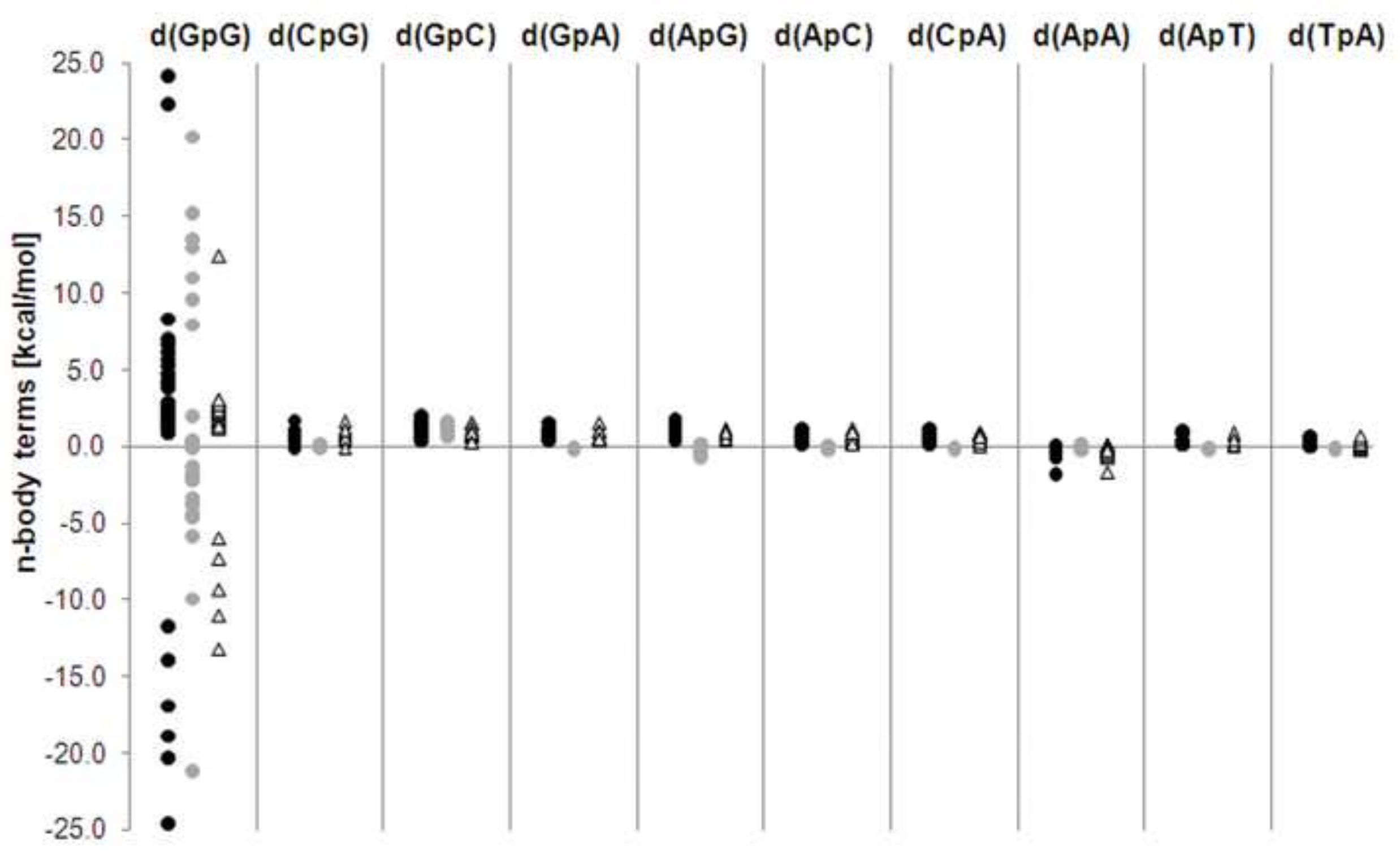




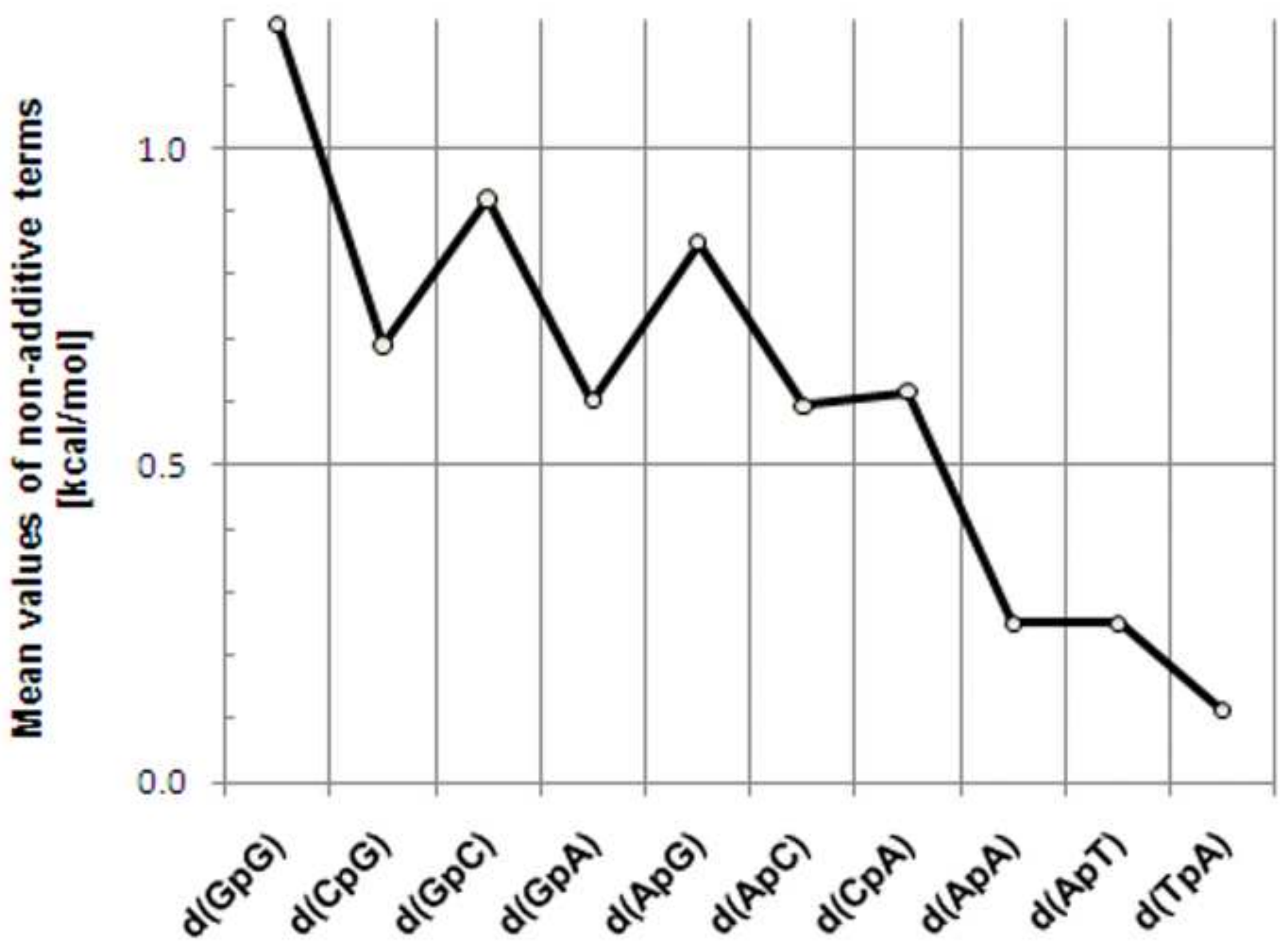




\section{additive $\Delta \mathrm{E}[\mathrm{kcal} / \mathrm{mol}]$}

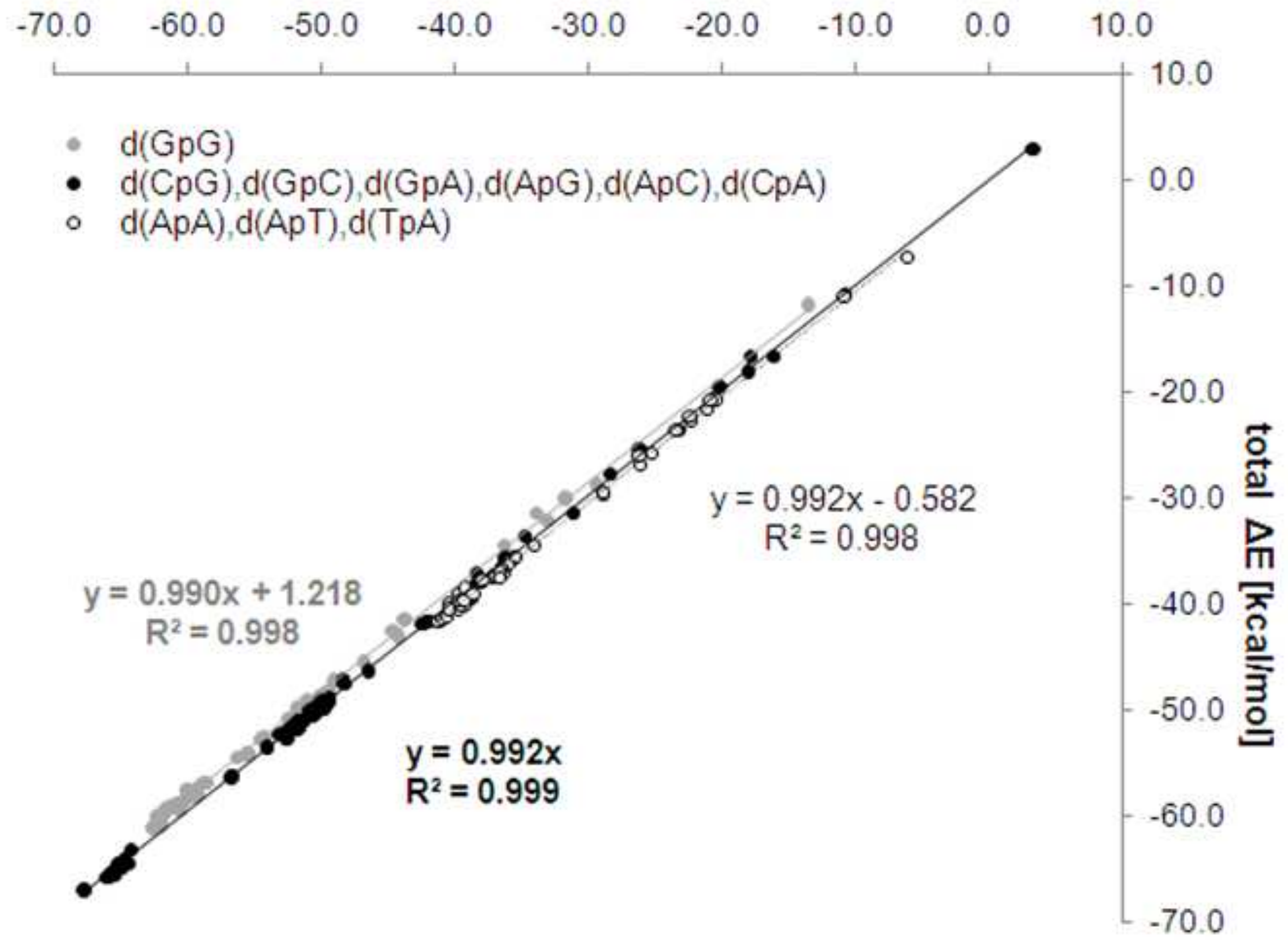




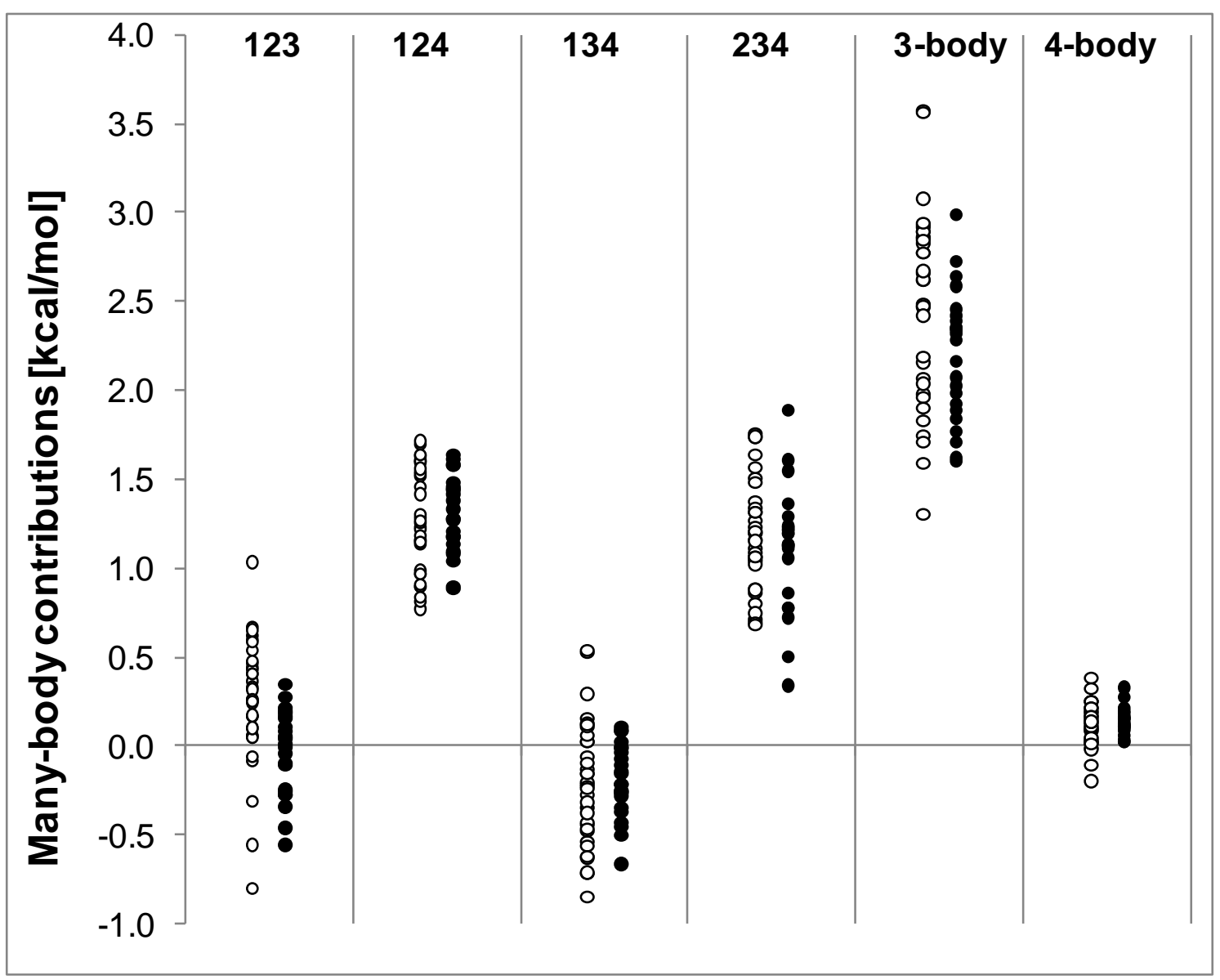

Figure 6. Distribution of many body contributions to intermolecular interaction in oxidized $\mathrm{d}(\mathrm{GpG})$ dinucleotide steps. Open circles denote $\mathrm{d}(\mathrm{GpZ})$ steps, while black circles stand for $\mathrm{d}(\mathrm{ZpG})$ sequence, where $\mathrm{Z}$ denotes 8-oxoguanine. 


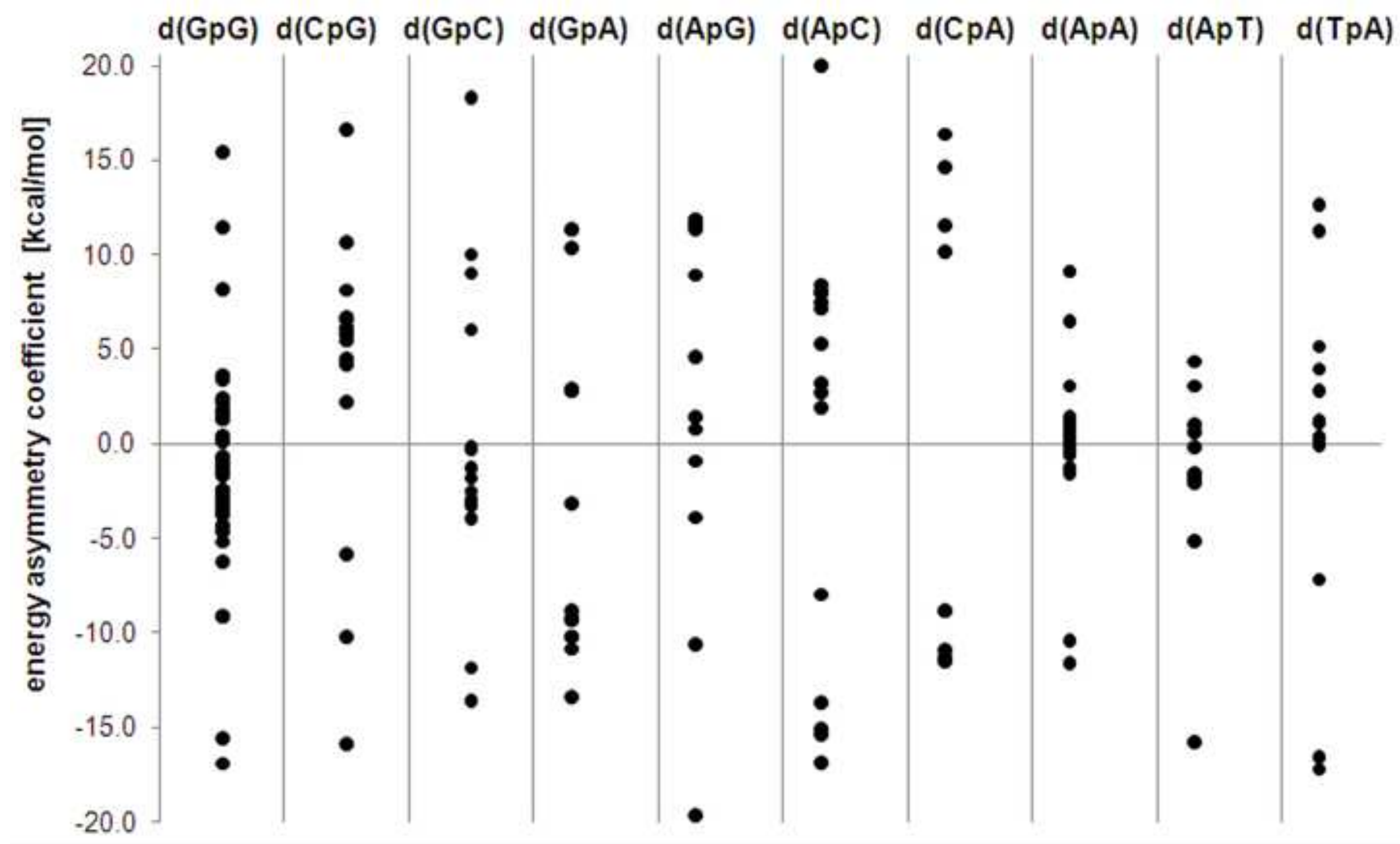




\section{Supplementary materials}

\section{Table S1}

The list of pdb files deposited in Nucleic Acid Database [33,34] that were used in this study as the source of structural parameters of dinucleotide steps. First two columns comprise B-DNA codes according to NDB and PDB identification styles. Resolution is provided in Angstroms. The final column comprises dinucleotide acronym along with the position of suitable base pairs in the B-DNA chain. If alternative pdb structures were used with to the same ID it is indicated in the bracket.

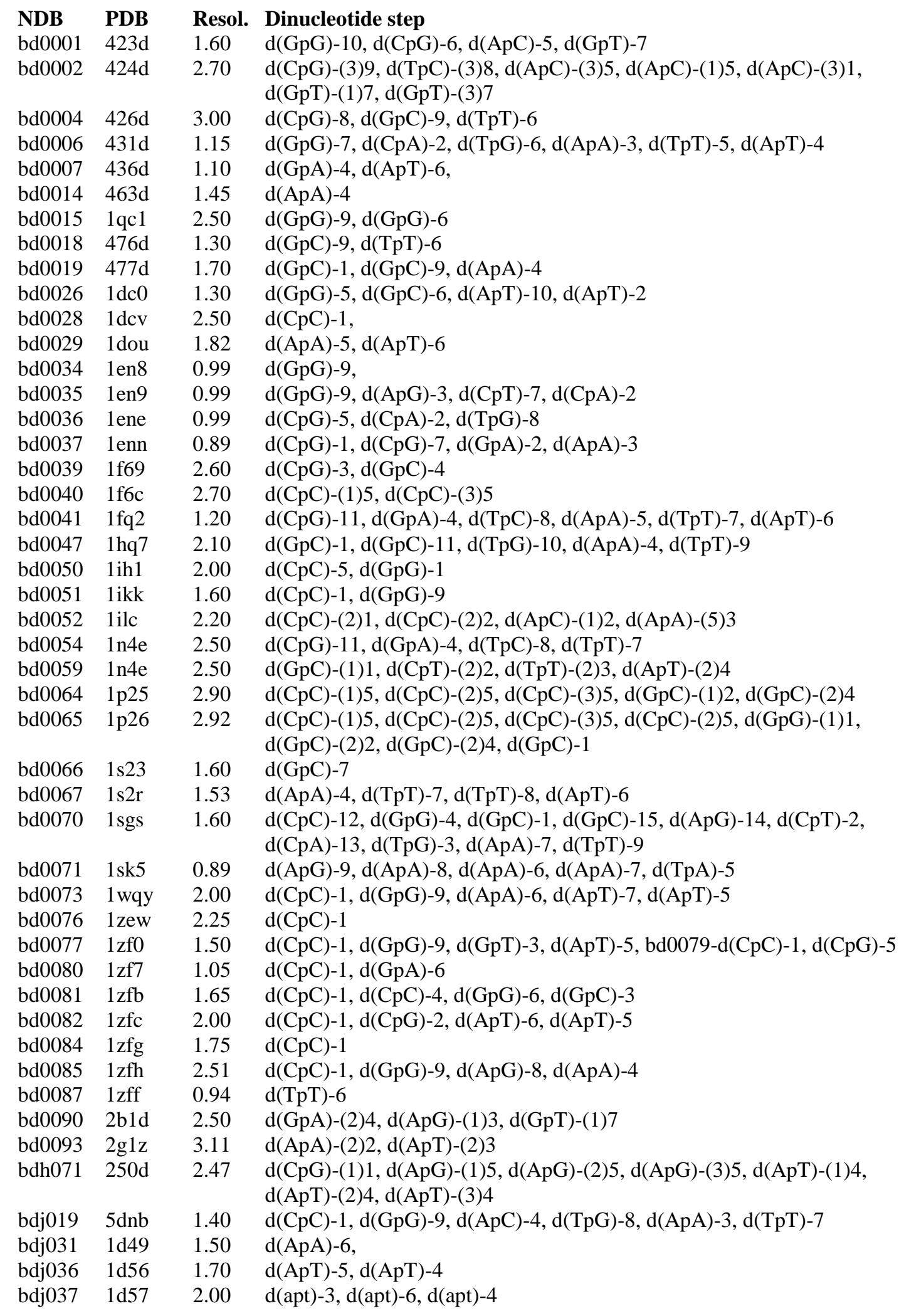




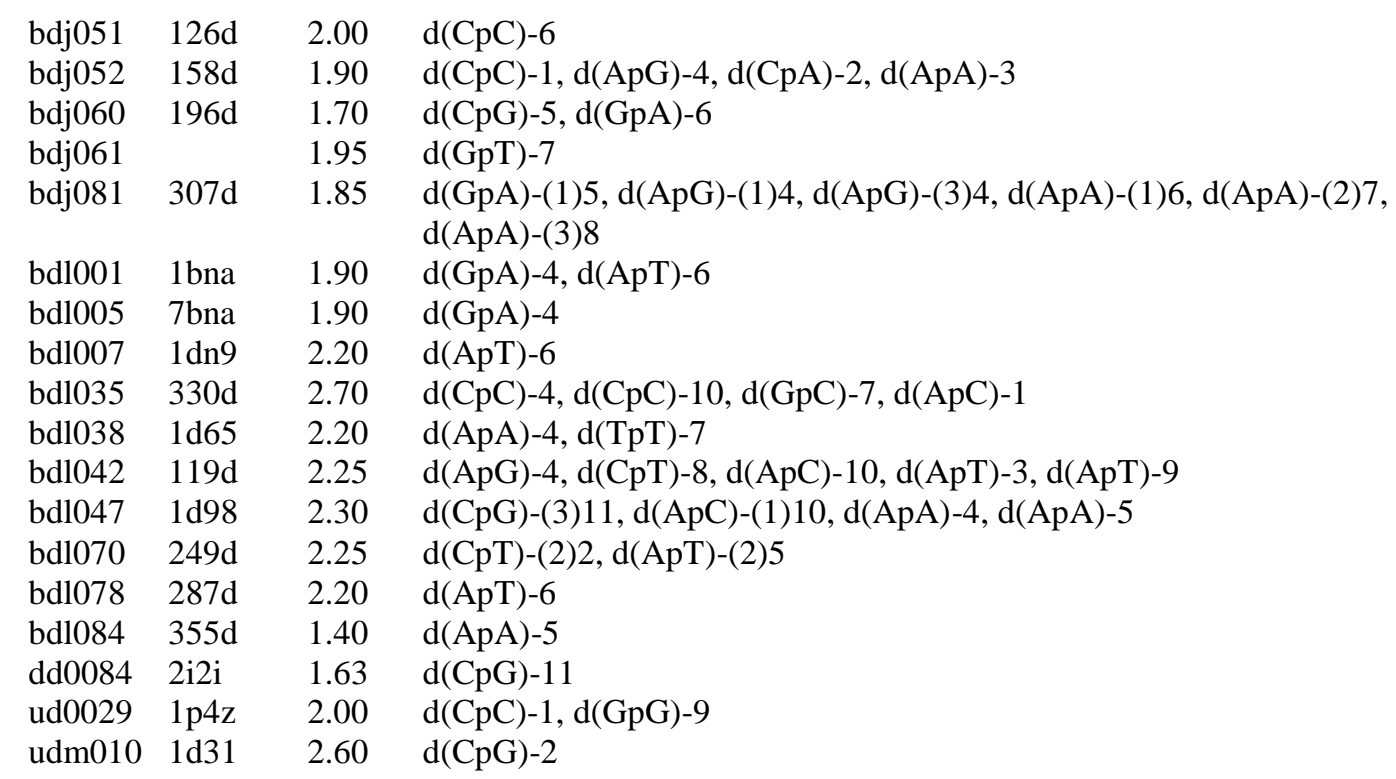


Figure 1. The schematic representation of intermolecular interactions in model $d(X p Y)$ dinucleotide steps. There are three distinct types of contacts, namely intra-strand stacking (5'$\left.\mathrm{X}_{1} / \mathrm{Y}_{2}-3^{\prime}, 5^{\prime}-\mathrm{Y}_{3}{ }_{3} / \mathrm{X}^{\prime}{ }_{4}-3^{\prime}\right)$, inter-strand stacking $\left(5^{\prime}-\mathrm{X}_{1}\left|\mathrm{Y}^{\prime}{ }_{3}-5^{\prime}, 3^{\prime}-\mathrm{Y}_{2}\right| \mathrm{X}_{4^{-}}{ }^{\prime}{ }^{\prime}\right)$ ) and hydrogen bonding $\left(5^{\prime}-\mathrm{X}_{1}-\mathrm{X}^{\prime}{ }_{4}{ }^{-} 3^{\prime}, 3^{\prime}-\mathrm{Y}_{2}-\mathrm{Y}^{\prime}{ }_{3}{ }^{-5}\right.$ '), where $\mathrm{X}, \mathrm{Y}$ denotes one of four nucleobases and $\mathrm{X}^{\prime}, \mathrm{Y}^{\prime}$ stands for corresponding complementary base. 


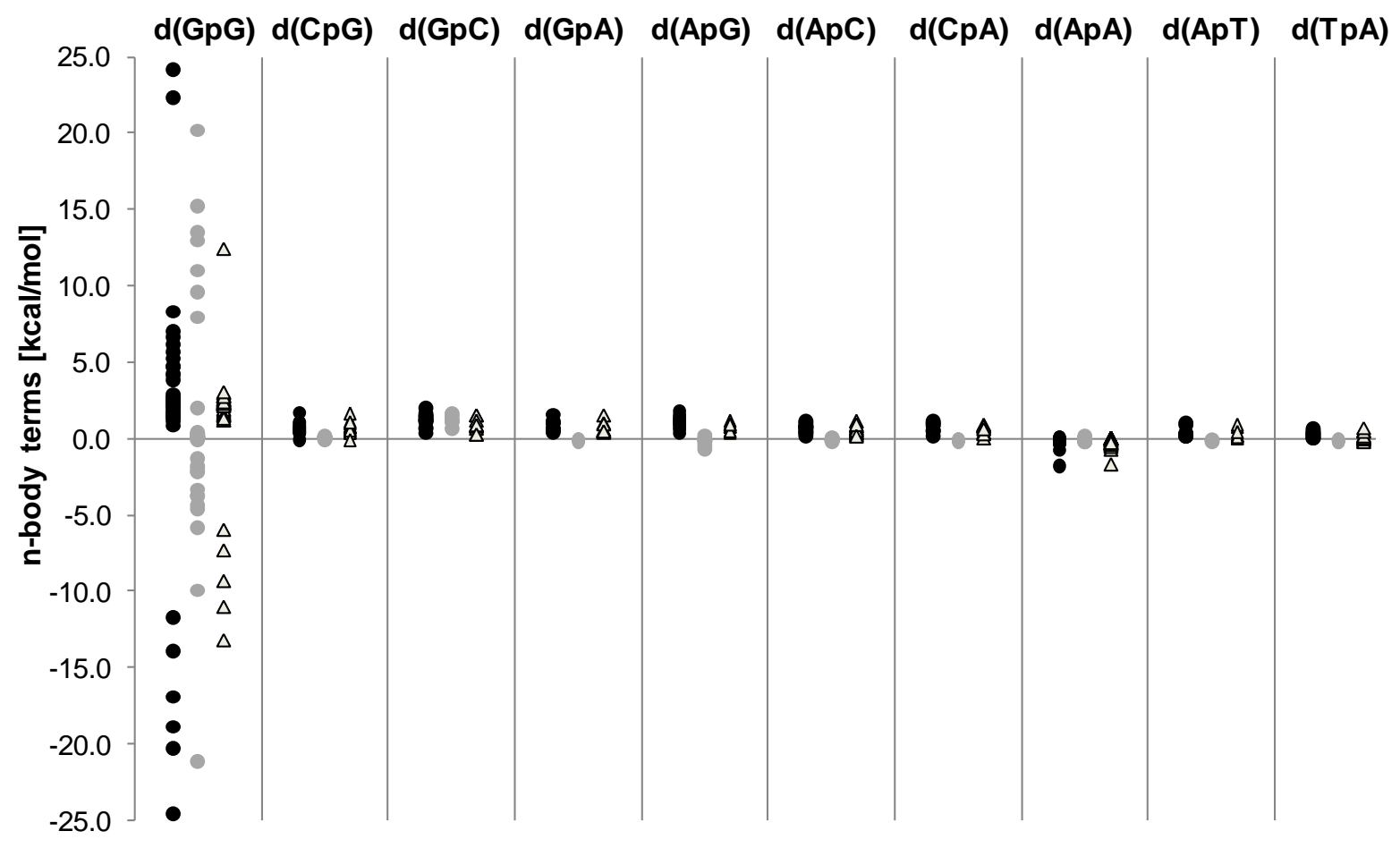

Fig. 2. The values of non-additive contributions to IIE energy of all ten possible model $\mathrm{d}(\mathrm{XpY})$ dinucleotide steps, where $\{(\mathrm{X}, \mathrm{Y}\}=\{\mathrm{A}, \mathrm{G}, \mathrm{C}, \mathrm{T}\}$. Black circles denote sum of all threebody contributions, gray circles correspond to four-body term and open triangles represent the sum of all non-additivities. 


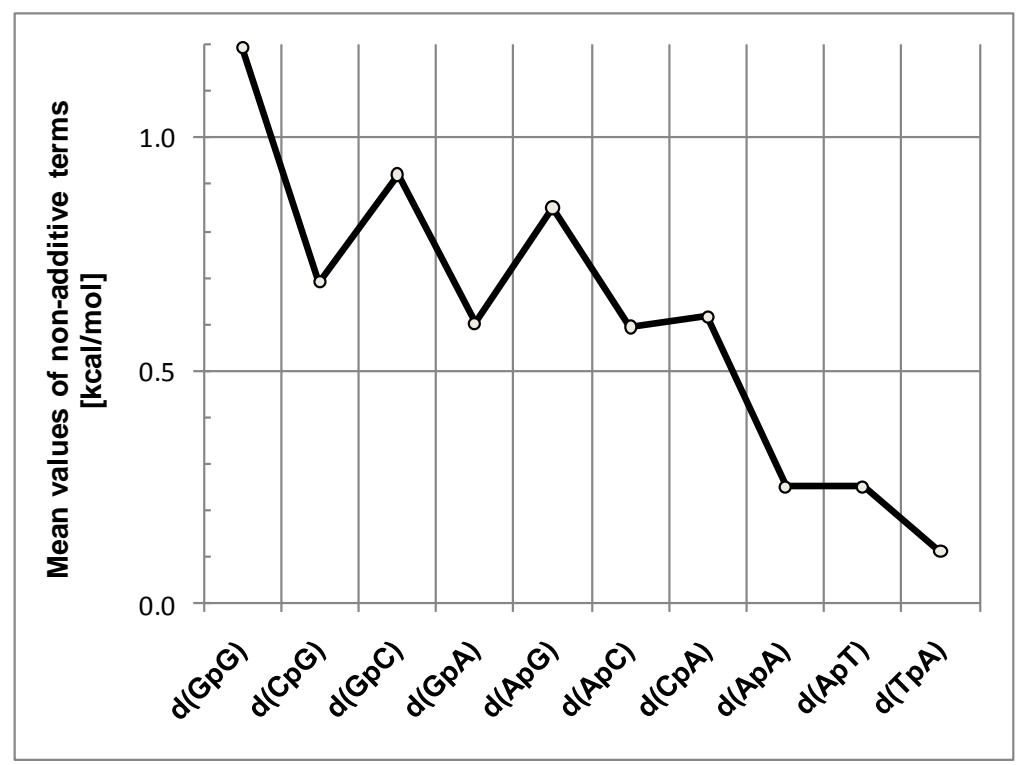

Fig. 3. The median values of all non additive terms estimated for ten possible dinucleotide steps. 


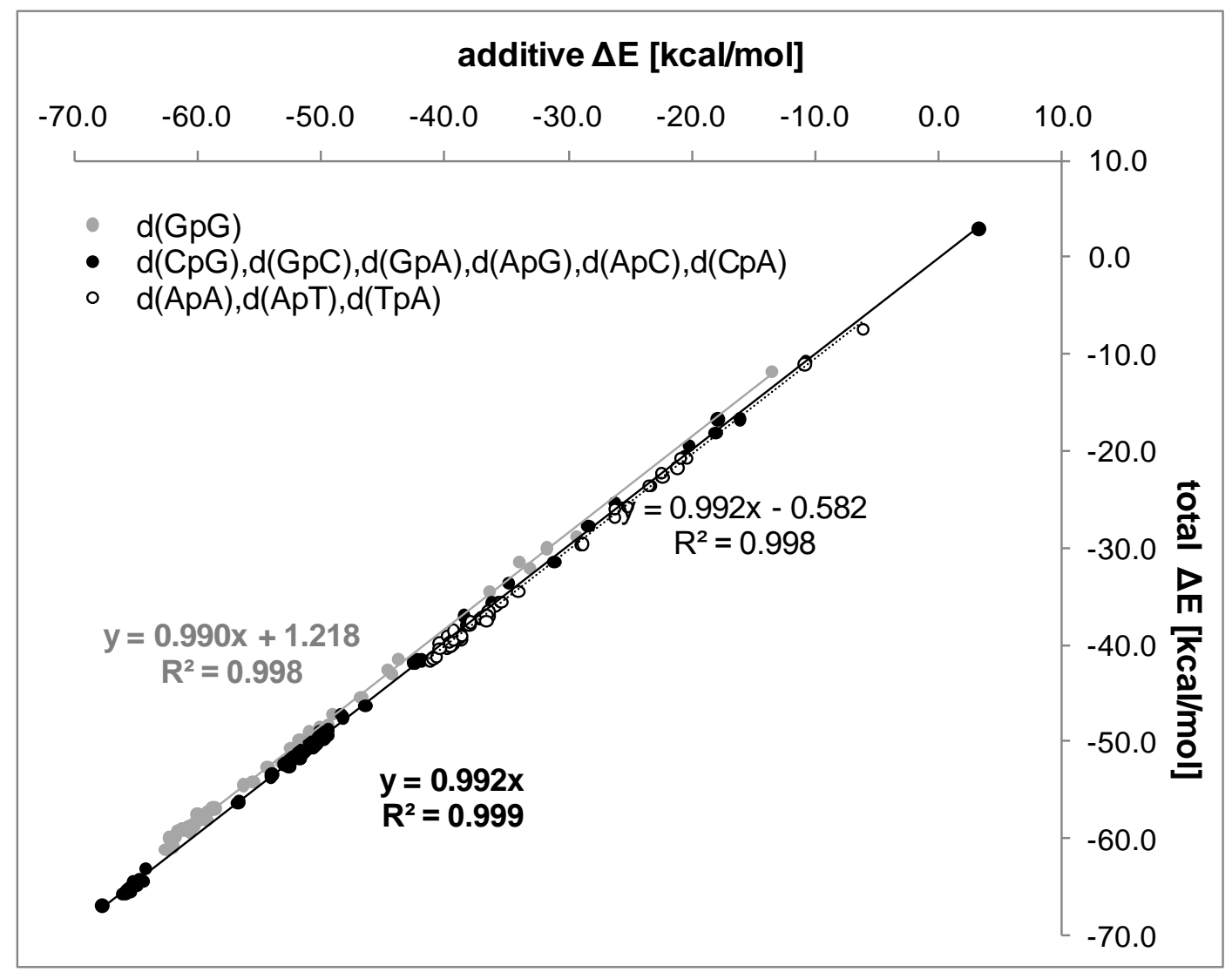

Fig.4. The correlation between full estimation of total intermolecular interaction energies in dinucleotide steps including all non-additive terms in tetramer basis set

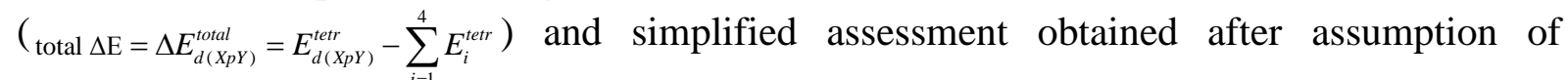
pairwise additivity of base-base interactions in dimer basis sets $\left(\right.$ additive $\left.\Delta \mathrm{E}=\Delta E_{d\left(X_{p} Y\right)}^{\text {add }}=\sum_{j>i=1}^{4}\left(E_{i j}^{d i m}-E_{i}^{d i m}-E_{j}^{d i m}\right)\right)$ 


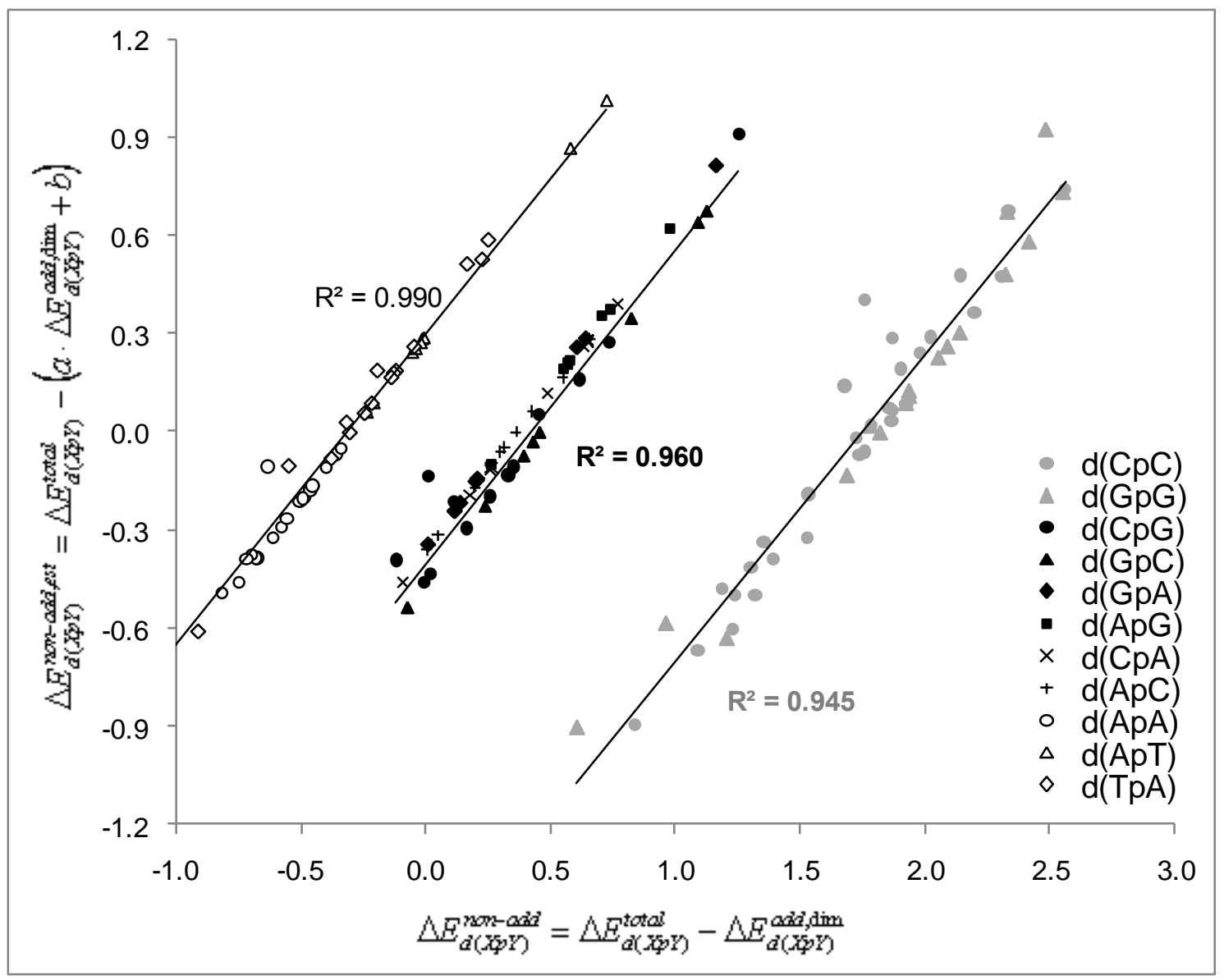

Fig.5. The applicability of linear relationships obtained in Fig.4 for estimation of non-additive contributions based on pair interactions estimated using dimer basis sets. 


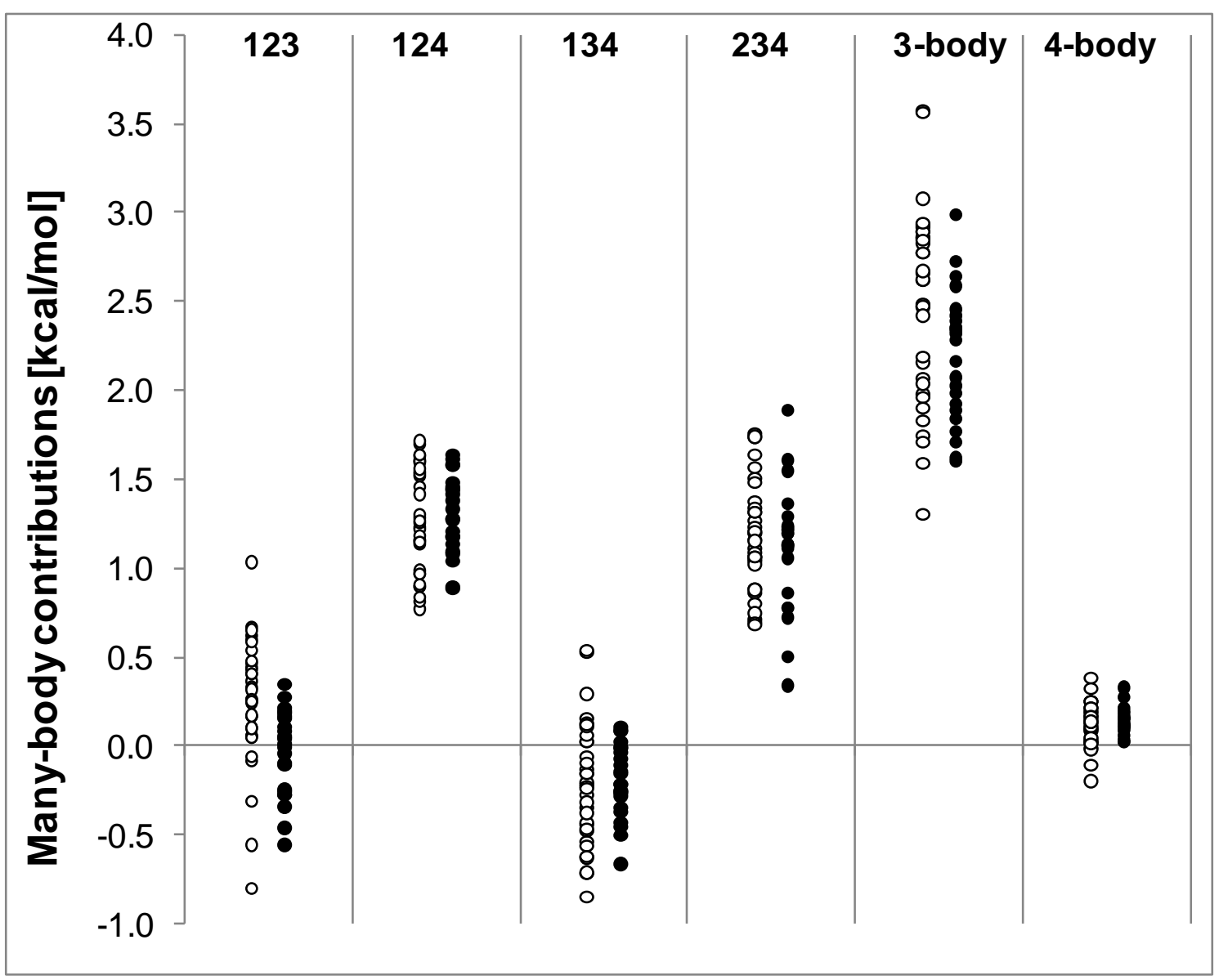

Figure 6. Distribution of many body contributions to intermolecular interaction in oxidized $\mathrm{d}(\mathrm{GpG})$ dinucleotide steps. Open circles denote $\mathrm{d}(\mathrm{GpZ})$ steps, while black circles stand for $\mathrm{d}(\mathrm{ZpG})$ sequence, where $\mathrm{Z}$ denotes 8-oxoguanine. 




Fig.7. The distribution of energy asymmetry coefficient $(\delta)$ estimated for all of analyzed dinucleotide steps. 